\title{
The Two-Parameter Odd Lindley Weibull Lifetime Model with Properties and Applications
}

\author{
Jehan. A. Almamy ${ }^{1}$, Mohamed Ibrahim ${ }^{2}$, M. S. Eliwa ${ }^{3}$, Saeed Al-mualim ${ }^{1,4}$ \& Haitham M. Yousof ${ }^{5}$ \\ ${ }^{1}$ Management Information System Department, Taibah University, Saudi Arabia \\ ${ }^{2}$ Department of Applied Statistics and Insurance, Faculty of Commerce, Damietta University, Damietta, Egypt \\ ${ }^{3}$ Department of Mathematics, Faculty of Science, Mansoura University, Egypt \\ ${ }^{4}$ Department of Statistics, Sana'a University, Yemen \\ ${ }^{5}$ Department of Statistics, Mathematics and Insurance, Benha University, Benha, Egypt \\ Correspondence: Haitham M. Yousof, Department of Statistics, Mathematics and Insurance, Benha University, Egypt.
}

Received: March 19, 2018 Accepted: March 27, 2018 Online Published: June 19, 2018

doi:10.5539/ijsp.v7n4p57

URL: https://doi.org/10.5539/ijsp.v7n4p57

\begin{abstract}
In this work, we study the two-parameter Odd Lindley Weibull lifetime model. This distribution is motivated by the wide use of the Weibull model in many applied areas and also for the fact that this new generalization provides more flexibility to analyze real data. The Odd Lindley Weibull density function can be written as a linear combination of the exponentiated Weibull densities. We derive explicit expressions for the ordinary and incomplete moments, moments of the (reversed) residual life, generating functions and order statistics. We discuss the maximum likelihood estimation of the model parameters. We assess the performance of the maximum likelihood estimators in terms of biases, variances, mean squared of errors by means of a simulation study. The usefulness of the new model is illustrated by means of two real data sets. The new model provides consistently better fits than other competitive models for these data sets. The Odd Lindley Weibull lifetime model is much better than Weibull, exponential Weibull, Kumaraswamy Weibull, beta Weibull, and the three parameters odd lindly Weibull with three parameters models so the Odd Lindley Weibull model is a good alternative to these models in modeling glass fibres data as well as the Odd Lindley Weibull model is much better than the Weibull, Lindley Weibull transmuted complementary Weibull geometric and beta Weibull models so it is a good alternative to these models in modeling time-to-failure data.
\end{abstract}

Keywords: Lindley distribution, Weibull distribution, Maximum likelihood, Moments, Order statistics

\section{Introduction}

The goal of this paper is to introduce a new two parameter alternative to the Weibull, beta Weibull, Lindley Weibull, exponential Weibull, Kumaraswamy Weibull, transmuted complementary Weibull geometric and the tree parameters Odd lindly Weibull (OLW) models that overcomes these mentioned drawbacks.

The probability density function (PDF) and CDF of the Weibull (W) distribution are given by (for $x \geq 0$ )

$$
g(x, \beta)=\beta x^{\beta-1} \exp \left(-x^{\beta}\right)
$$

and

$$
G(x, \beta)=1-\exp \left(-x^{\beta}\right),
$$

respectively, where $\beta>0$ is a shape parameter. Some useful generalization of the Weibull distribution studied in the literature includes, but are not limited to, Mudholkar and Srivastava (1993), Mudholkar et al. (1995), Mudholkar et al. (1996), Xie and Lai (1995), Ghitany et al. (2005), Famoye et al. (2005), Sarhan and Zaindin (2009), Silva et al. (2010), Aryal and Tsokos (2011), Xie et al. (2002), Lai et al. (2003), Cordeiro et al. (2010), Provost et al. (2011), Cordeiro et al. (2012), Shahbaz et al. (2012), Khan and King (2013), Cordeiro et al. (2013), Merovci and Elbatal (2013), Hanook et al. (2013), Yousof et al. (2015), Cordeiro et al. (2014), Lee et al. (2007), Elbatal and Aryal (2013), Aryal and Elbatl (2015), Afify et al. (2016), Nofal et al. (2016), El-Bassiouny et al. (2016), Yousof et al. (2017a,b,c,d), Aryal et al. (2017a,b), Korkmaz et al. (2017), El-Bassiouny et al. (2017), Alizadeh et al. (2017a,b), Brito et al. (2015). Alizadeh et al. (2018), Yousof et al. (2018), Cordeiro et al. (2018), Hamedani et al. (2018), among others. A state-of-the-art survey on the class of such generalized Weibull distributions can be found in Lai et al. (2001) and Nadarajah (2009)

The probability density function (PDF) and CDF of the OL-G family of distribution (Silva et al. (2017)) are given by

$$
f(x ; \alpha, \xi)=\alpha^{2}(1+\alpha)^{-1} g(x ; \xi) \bar{G}(x ; \xi)^{-3} \exp [-\alpha G(x ; \xi) / \bar{G}(x ; \xi)],
$$


and

$$
F(x ; \alpha, \xi)=1-[\alpha+\bar{G}(x ; \xi)](1+\alpha)^{-1} \bar{G}(x ; \xi)^{-1} \exp [-\alpha G(x ; \xi) / \bar{G}(x ; \xi)],
$$

respectively. To this end, by using equations (1), (2) and (3) to obtain the two-parameter OLW PDF (for $x \geq 0$ ). A random variable $\mathrm{X}$ is said to have the OLW distribution if its density function and CDF are given by

$$
f(x ; \alpha, \beta)=\alpha^{2}(1+\alpha)^{-1} \beta x^{\beta-1}\left[\exp \left(2 x^{\beta}\right)\right] \exp \left[-\alpha \frac{1-\exp \left(-x^{\beta}\right)}{\exp \left(-x^{\beta}\right)}\right],
$$

and

$$
F(x ; \alpha, \beta)=1-(1+\alpha)^{-1}\left[\alpha+\exp \left(-x^{\beta}\right)\right] \exp \left(x^{\beta}\right) \exp \left\{-\alpha\left[\exp \left(x^{\beta}\right)-1\right]\right\},
$$

respectively, we write $X \sim \operatorname{OLW}(\alpha, \beta)$, where $\alpha$ is a positive shape parameter. The PDF in (5) and the CDF in (6) are firstly introduced by Silva et al. (2017). Henceforth, the PDF of $X$ in (5) can be easily expressed as

$$
f(x)=\sum_{i, k=0}^{\infty} v_{i, k} g(x ;(i+k+1), \beta),
$$

where

$$
v_{i, k}=(-1)^{k} \alpha^{2+k}(\alpha+1)^{-1}[(i+k+1) i !]^{-1} \Gamma(i+k+3) / \Gamma(k+3),
$$

and $g(x ; \delta, \beta)$ is PDF of Exp-W model with positive parameters $\delta$ and $\beta$. A handbook, by Rinne (2009), covers the Weibull model in many of its aspects. The study of the family of Exp-W models and their applications attracted the interest of researchers in the nineties. Such interest is growing since then. The CDF of $X$ can be given by integrating (7) as

$$
F(x)=\sum_{i, k=0}^{\infty} v_{i, k} G(x ;(i+k+1), \beta),
$$

where $G(x ; \delta, \beta)$ is PDF of Exp-W model with positive parameters $\delta$ and $\beta$. For further information about the Exp-W distribution we refer to Mudholkar and Srivastava (1993) and Nadarajah and Kotz (2006). For more details about the OL-G family and its properties see Silva et al. (2017).
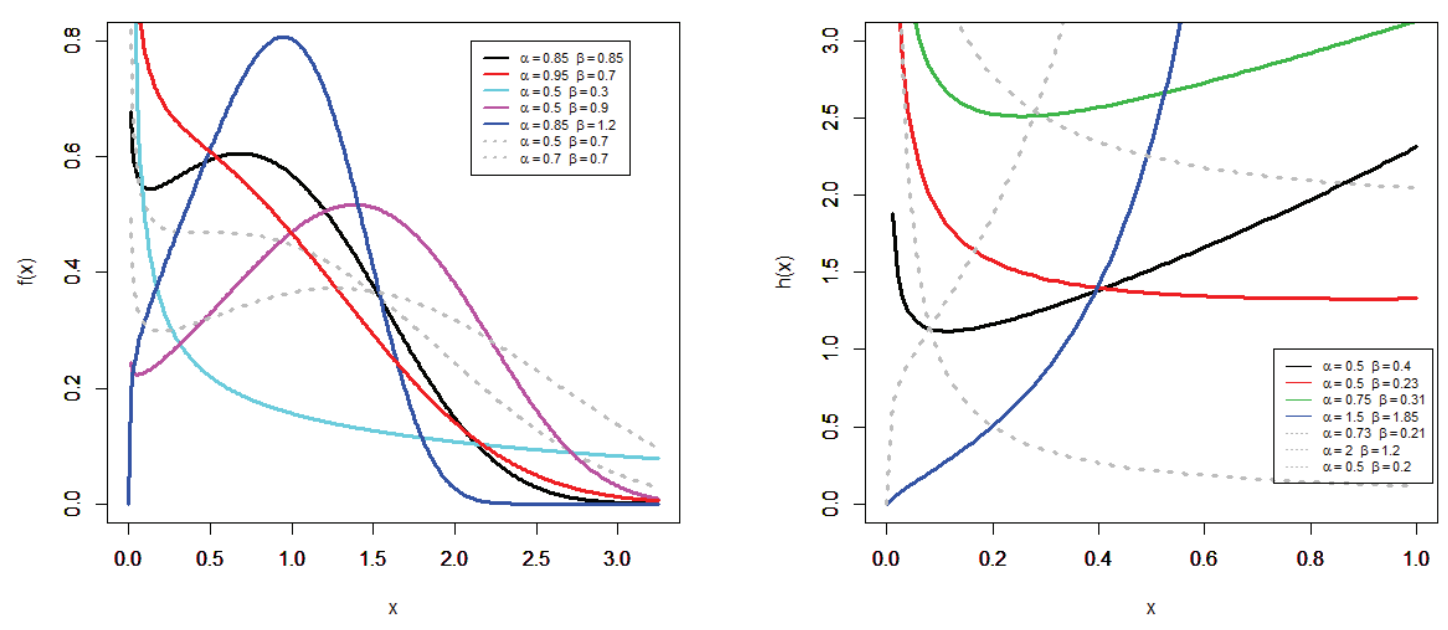

Figure 1. Plots of the OLW PDF and HRF for some parameter values.

The justification for the practicality of the OLW lifetime model is based on the wider use of the W model. Aswell as we are motivated to introduce the OLW lifetime model because it exhibits increasing, decreasing as well as bathtub hazard rates as illustrated in Figure 2. It is shown in Section 1 that the OLW lifetime model can be viewed as a mixture of the 
two-parameter Exp-W distributions introduced by Mudholkar and Srivastava (1993) and Mudholkar et al. (1995). It can be viewed as a suitable model for fitting the unimodal and left skewed data. The OLW lifetime model is much better than Weibull, exponential Weibull, Kumaraswamy Weibull, beta Weibull, and the three parameters Odd lindly Weibull with three parameters models so the OLW lifetime model is a good alternative to these models in modeling glass fibres data as well as the OLW lifetime model is much better than the Weibull, Lindley Weibull transmuted complementary Weibull geometric and beta Weibull models so the OLW lifetime model is a good alternative to these models in modeling time-to-failure data.

\section{Statistical Properties}

\subsection{Quantile Functions}

Let $X$ be an arbitrary random variable (r.v.) with $\operatorname{CDF} F(x ; \alpha, \beta)$. For any $u \in(0.1)$, the $\mathrm{u}^{\text {th }}$ quantile function (QF) $Q(u)$ of the r.v. $X$ is the solution of $u=F(Q(u))$ for all $Q(u)>0$, from Equation (6), we get

$$
(u-1)(1+\alpha) \exp (1+\alpha)=-\frac{1+\alpha-G(Q(u))}{1-G(Q(u))} \exp \left\{-\frac{1+\alpha-G(Q(u))}{1-G(Q(u))}\right\},
$$

where

$$
-\frac{1+\alpha-G(Q(u))}{1-G(Q(u))}
$$

is the Lambert $W(\cdot)$ function of the real argument $(u-1)(1+\alpha) \exp (1+\alpha)$. From Silva et al. (2017), we can write the following equation for QF of the OLW model

$$
Q(u)=\left[1-\log \left(1-\left\{1+\alpha\left[1+W_{-1}((u-1)(1+\alpha) \exp (1+\alpha))^{-1}\right]\right\}\right)\right]^{\frac{1}{\beta}},
$$

where $W(\cdot)$ is Lambert function.

\subsection{Moments}

The $r^{\text {th }}$ ordinary moment of $X$ is given by $\mu_{r}^{\prime}=\int_{0}^{\infty} x^{r} f(x) d x=E\left(X^{r}\right)$. Using (7), we get

$$
\mu_{r}^{\prime}=\sum_{i, j, k=0}^{\infty} v_{i, k} \Upsilon_{j}^{(i+k+1)} \prod_{m=0}^{\frac{r}{\beta}-1}\left(\frac{r}{\beta}-m\right), \forall r>-\beta,
$$

where

$$
\begin{gathered}
\Upsilon_{j}^{(\omega, r)}=\omega(-1)^{i}(j+1)^{-(r+\beta) / \beta}\left(\begin{array}{c}
\omega-1 \\
j
\end{array}\right), \\
\prod_{m=0}^{v-1}(v-m)=\Gamma(1+v)=v(v-1)(v-2) \ldots 1, v \in \mathbb{R}^{+}
\end{gathered}
$$

and $\Gamma(\zeta)=\int_{0}^{\infty} x^{\zeta-1} e^{-t} d x$ is the complete gamma function. The $r^{t h}$ incomplete moment of $X$, say $\varphi_{r}(t)$, is given by $\varphi_{r}(t)=\int_{0}^{t} x^{r} f(x) d x$. Using Equation (7), we obtain

$$
\varphi_{r}(t)=\gamma\left(1+r \beta^{-1}, t^{-\beta}\right) \sum_{i, j, k=0}^{\infty} v_{i, k} \Upsilon_{j}^{(i+k+1, r)}, \forall r>-\beta,
$$

where $\gamma(\zeta, x)=\int_{0}^{x} x^{\zeta-1} e^{-x} d x$ is the incomplete gamma function.

\subsection{Order Statistics and Their Moments}

Let $X_{1}, \ldots, X_{n}$ be a random sample from the OLW model of distributions and let $X_{1: 1}, \ldots, X_{n: n}$ be the corresponding order statistics. The PDF of the $i^{t h}$ order statistic, say $X_{i: n}$, can be expressed as

$$
f_{i: n}(x)=[\mathrm{B}(i, n-i+1)]^{-1} f(x) F(x)^{i-1}[1-F(x)]^{n-i},
$$

where $B(\cdot, \cdot)$ is the beta function. Substituting (5) and (6) in Equation (11), we obtain

$$
f_{i: n}(x)=\sum_{m, p=0}^{\infty} \sum_{j=0}^{k+n-i} v_{j, m, p} g(x ;(j+m+p+1), \beta),
$$


where

$$
\begin{aligned}
v_{j, m, h}= & \sum_{k=0}^{i-1}(-1)^{k+m} \alpha^{j+m+2}(1+\alpha)^{-(j+1)}[m !(j+m+p+1)]^{-1} \\
& \times[\mathrm{B}(i, n-i+1)]^{-1}\left(\begin{array}{c}
j+m+p \\
j+m
\end{array}\right)\left(\begin{array}{c}
k+n-1 \\
j
\end{array}\right)\left(\begin{array}{c}
i-1 \\
k
\end{array}\right) .
\end{aligned}
$$

Then, the $q^{\text {th }}$ moment of $X_{i: n}$ is given by

$$
E\left(X_{i: n}^{q}\right)=\sum_{m, p, h=0}^{\infty} \sum_{j=0}^{k+n-i} v_{j, m, p} \Upsilon_{h}^{(j+m+p+1, q)} \prod_{w=0}^{\frac{q}{\beta}-1}\left(\frac{q}{\beta}-w\right), \forall q>-\beta .
$$

Based upon the moments in Equation (12), we can derive explicit expressions for the L-moments of $X$ as infinite weighted linear combinations of the means of suitable OLW order statistics. They are linear functions of expected order statistics defined by

$$
\lambda_{r}=r^{-1} \sum_{d=0}^{r-1}(-1)^{d}\left(\begin{array}{c}
r-1 \\
d
\end{array}\right) E\left(X_{r-d: r}\right), r \geq 1 .
$$

\subsection{Moment of Residual and Reversed Residual Lifes}

The $n^{\text {th }}$ moment of the residual life, say $z_{n}(t)=E\left[(X-t)^{n} \mid X>t\right], n=1,2, \ldots$, uniquely determines $F(x)$. The $n^{t h}$ moment of the residual life of $X$ is given by

$$
z_{n}(t)=\frac{\int_{t}^{\infty}(x-t)^{n} d F(x)}{1-F(t)}
$$

We can write

$$
\begin{aligned}
z_{n}(t) & =[1-F(t)]^{-1} \sum_{i, k=0}^{\infty} \sum_{r=0}^{n} v_{i, k}(-t)^{n-r}\left(\begin{array}{l}
n \\
r
\end{array}\right) \int_{t}^{\infty} x^{r} g(x ;(i+k+1), \beta) d x \\
& =\gamma\left(1+n \beta^{-1}, t^{-\beta}\right)[1-F(t)]^{-1} \sum_{i, j, k=0}^{\infty} \sum_{r=0}^{n} \Upsilon_{i, j, k, r}^{(i+k+1, n)}, \forall n>-\beta,
\end{aligned}
$$

where

$$
\Upsilon_{i, j, k, r}^{(i+k+1, n)}=v_{i, k} t^{n-r}(i+k+1)(-1)^{i+n-r}(j+1)^{-(n+\beta) / \beta}\left(\begin{array}{c}
i+k \\
j
\end{array}\right)\left(\begin{array}{l}
n \\
r
\end{array}\right) .
$$

The $n^{\text {th }}$ moment of the reversed residual life, say $Z_{n}(t)=E\left[(t-X)^{n} \mid X \leq t\right]$, for $t>0$ and $n=1,2, \ldots$, uniquely determines $F(x)$. We have

$$
Z_{n}(t)=\frac{\int_{0}^{t}(t-x)^{n} d F(x)}{F(t)} .
$$

Then, the $n^{\text {th }}$ moment of the reversed residual life of $X$ becomes

$$
\begin{aligned}
Z_{n}(t) & =F(t)^{-1} \sum_{i, k=0}^{\infty} \sum_{r=0}^{n} v_{i, k}(-1)^{r}\left(\begin{array}{l}
n \\
r
\end{array}\right) t^{n-r} \int_{0}^{t} x^{r} g(x ;(i+k+1), \beta) d x \\
& =\gamma\left(1+n \beta^{-1}, t^{-\beta}\right) F(t)^{-1} \sum_{i, j, k=0}^{\infty} \sum_{r=0}^{n} \Omega_{i, j, k, r}^{(i+k+1, n)}, \forall n>-\beta,
\end{aligned}
$$

where

$$
\Omega_{i, j, k, r}^{(i+k+1, n)}=v_{i, k} t^{n-r}(i+k+1)(-1)^{i+r}(j+1)^{-(n+\beta) / \beta}\left(\begin{array}{c}
i+k \\
j
\end{array}\right)\left(\begin{array}{l}
n \\
r
\end{array}\right) .
$$




\section{Maximum Likelihood Method}

We consider the estimation of the unknown parameters of the OLW model from complete samples only by maximum likelihood method. The MLEs of the parameters of the $\operatorname{OLW}(\alpha, \beta)$ model is now discussed. Let $x_{1}, \ldots, x_{n}$ be a random sample of this distribution with parameter vector $\boldsymbol{\Psi}=(\alpha, \beta)^{\top}$. The log-likelihood function for $\boldsymbol{\Psi}$, say $\ell=\ell(\boldsymbol{\Psi})$, is given by

$$
\ell=\ell(\boldsymbol{\Psi})=2 n \log (\alpha)-n \log (1+\alpha)+n \log \beta+(\beta-1) \sum_{i=1}^{n} \log \left(x_{i}\right)+2 \sum_{i=1}^{n} x_{i}^{\beta}-\alpha \sum_{i=1}^{n} \frac{1-\exp \left(-x_{i}^{\beta}\right)}{\exp \left(-x_{i}^{\beta}\right)},
$$

the last equation can be maximized either by using the different programs like R (optim function), SAS (PROC NLMIXED) or by solving the nonlinear likelihood equations obtained by differentiating Equation 13. The score vector elements, $\mathbf{U}(\boldsymbol{\Psi})=\frac{\partial \ell}{\partial \Psi}=\left(\frac{\partial \ell}{\partial \alpha}, \frac{\partial \ell}{\partial \beta}\right)^{\top}$ can be easely obtained, we can obtain the estimates of the unknown parameters by setting the score vector to zero, $\mathbf{U}(\widehat{\boldsymbol{\Psi}})=\mathbf{0}$. Solving these equations simultaneously gives the MLEs $\widehat{\alpha}$ and $\widehat{\beta}$. For the OLW distribution, all the second order derivatives exist. The interval estimation of the model parameters requires the $2 \times 2$ observed information matrix $J(\boldsymbol{\Psi})=\left\{J_{i j}\right\}$ for $i, j=\alpha, \beta$. The multivariate normal $N_{2}\left(0, J(\widehat{\mathbf{\Psi}})^{-1}\right)$ distribution, under standard regularity conditions, can be used to provide approximate confidence intervals for the unknown parameters, where $J(\widehat{\boldsymbol{\Psi}})$ is the total observed information matrix evaluated at $\widehat{\boldsymbol{\Psi}}$. Then, approximate $100(1-\delta) \%$ confidence intervals for $\alpha$ and $\beta$ can be determined by: $\widehat{\alpha} \pm z_{\delta / 2} \sqrt{\widehat{J}_{\alpha \alpha}}$ and $\widehat{\beta} \pm z_{\delta / 2} \sqrt{\widehat{J}_{\beta \beta}}$, where $z_{\delta / 2}$ is the upper $\delta^{\text {th }}$ percentile of the standard normal model. Further works could be addressed using different methods to estimate the OLW parameters such as least squares, moments, weighted least squares, Jackknife, Cram'er-von-Mises, bootstrap, Bayesian analysis, AndersonDarling, among others, and compare the estimators based on these methods.

\section{Simulation Studies}

We consider a random sample of size $\mathrm{n}=50,150,200,250,300$ and 500 from our density corresponding to particular choices of the parameters as follows: $\alpha=0.25, \beta=0.5, \alpha=1.5, \beta=0.9$ and $\alpha=0.8, \beta=1.5$, the results are presented in Tables 1,2 and 3 respectively. Below we provide the MLEs, biases (Bias), variances (Var), mean square of errors (MSEs) and Confidence Interval for the estimates of all the parameters under both the methods of estimation. The log-likelihood function can be maximized directly via the R-package or by solving the nonlinear likelihood equations obtained by differentiating the PDF (5) (using the optim function as well as the Max-BFGS subroutines. One can observe the estimates of the unknown parameters by setting the score vector to zero, and then using any statistical software to solve them numerically. The results show that the maximum likelihood estimation performs well. In general, the biases, variances and MSEs of the parameters are reasonably small. The biases, variances and MSEs always decrease as the sample size increases. The results suggest that the maximum likelihood method can be used to estimate the parameters of the OLW model.

Table 1 provides the biases, variances, MSEs and Confidence Interval under the method of maximum likelihood. We consider 1000 simulations for drawing random samples each of size $n=50,150,200,250,300$ and 500 drawn from our density respectively when $\alpha=0.25$ and $\beta=0.5$.

Table 1. The MLEs, Bias, Var, and MSE values for the OLWD

\begin{tabular}{|c|c|c|c|c|c|}
\hline $\mathrm{n}$ & Parameter (MLE) & Bias & Var & MSE & Confidence Interval \\
\hline \multirow[t]{2}{*}{50} & $\alpha=0.25(0.247229)$ & -0.002771 & 0.0017944 & 0.001802 & (0.1691,0.3366) \\
\hline & $\beta=0.5(0.507311)$ & 0.007311 & 0.0011746 & 0.001228 & $(0.4418,0.5759)$ \\
\hline \multirow[t]{2}{*}{150} & $\alpha=0.25(0.247489)$ & -0.002511 & 0.0006301 & 0.000636 & $(0.1993,0.2985)$ \\
\hline & $\beta=0.5(0.503367)$ & 0.003367 & 0.0003829 & 0.000394 & $(0.4677,0.5436)$ \\
\hline \multirow[t]{2}{*}{200} & $\alpha=0.25(0.248688)$ & -0.001312 & 0.0004553 & 0.000457 & $(0.2072,0.2918)$ \\
\hline & $\beta=0.5(0.502525)$ & 0.002525 & 0.0002828 & 0.000289 & $(0.4699,0.5368)$ \\
\hline \multirow[t]{2}{*}{250} & $\alpha=0.25(0.249041)$ & -0.000959 & 0.0003657 & 0.000367 & $(0.2148,0.2886)$ \\
\hline & $\beta=0.5(0.501742)$ & 0.001742 & 0.000216 & 0.000219 & $(0.4726,0.5316)$ \\
\hline \multirow[t]{2}{*}{300} & $\alpha=0.25(0.249389)$ & -0.000611 & 0.00032 & 0.00032 & $(0.2155,0.2861)$ \\
\hline & $\beta=0.5(0.501331)$ & 0.001331 & 0.0001873 & 0.000189 & $(0.4743,0.5301)$ \\
\hline \multirow[t]{2}{*}{500} & $\alpha=0.25(0.249847)$ & -0.000153 & 0.0001874 & 0.000187 & $(0.2245,0.2787)$ \\
\hline & $\beta=0.5(0.500251)$ & 0.000251 & 0.0001117 & 0.000112 & $(0.4798,0.5218)$ \\
\hline
\end{tabular}


Table 2 provides the MLEs, biases, variances, MSEs and Confidence Interval under the method of maximum likelihood. We consider 1000 simulations for drawing random samples each of size $n=50,150,200,250,300$ and 500 drawn from our density respectively when $\alpha=1.5$ and $\beta=0.9$.

Table 2. The MLEs, Bias, Var, and MSE values for the OLWD

\begin{tabular}{cccccc}
\hline \hline $\mathrm{n}$ & Parameter(MLE) & Bias & Var & MSE & Confidence Interval \\
\hline \hline \multirow{2}{*}{50} & $\alpha=1.5(1.529613)$ & 0.029613 & 0.033058 & 0.033935 & $(1.2203,1.9334)$ \\
\cline { 2 - 6 } & $\beta=0.9(0.929152)$ & 0.029152 & 0.0156265 & 0.016476 & $(0.7296,1.2245)$ \\
\hline \multirow{2}{*}{50} & $\alpha=1.5(1.51163)$ & 0.01163 & 0.0096352 & 0.00977 & $(1.3240,1.7228)$ \\
\cline { 2 - 6 } & $\beta=0.9(0.907027)$ & 0.007027 & 0.0038567 & 0.003906 & $(0.7955,1.0367)$ \\
\hline \multirow{2}{*}{200} & $\alpha=1.5(1.508664)$ & 0.008664 & 0.0077297 & 0.007805 & $(1.3531,1.6969)$ \\
\cline { 2 - 6 } & $\beta=0.9(0.906696)$ & 0.006696 & 0.0031 & 0.003145 & $(0.8050,1.0131)$ \\
\hline \multirow{2}{*}{300} & $\alpha=1.5(1.503555)$ & 0.003555 & 0.0056863 & 0.005699 & $(1.3631,1.6487)$ \\
\cline { 2 - 6 } & $\beta=0.9(0.905057)$ & 0.005057 & 0.0023803 & 0.002406 & $(0.8178,1.0088)$ \\
\cline { 2 - 6 } & $\beta=0.9(0.904546)$ & 0.004546 & 0.0020092 & 0.00203 & $(0.8215,0.9957)$ \\
\hline \multirow{2}{*}{500} & $\alpha=1.5(1.502812)$ & 0.002812 & 0.0029378 & 0.002946 & $(1.4031,1.6138)$ \\
\cline { 2 - 6 } & $\beta=0.9(0.903687)$ & 0.003687 & 0.001307 & 0.001321 & $(0.8385,0.9740)$ \\
\hline \hline
\end{tabular}

Table 3 provides the biases, variances, MSEs and Confidence Interval under the method of maximum likelihood. We consider 1000 simulations for drawing random samples each of size $n=50,150,200,250,300$ and 500 drawn from our density respectively when $\alpha=0.8$ and $\beta=1.5$.

Table 3. The MLEs, Bias, Var, and MSE values for the OLWD

\begin{tabular}{cccccc}
\hline \hline $\mathrm{n}$ & Parameter (MLE) & Bias & Var & MSE & Confidence Interval \\
\hline \hline 50 & $\alpha=0.8(0.80632)$ & 0.00632 & 0.0091889 & 0.009229 & $(0.6242,1.0034)$ \\
\cline { 2 - 6 } & $\beta=1.5(1.538177)$ & 0.038177 & 0.0255296 & 0.026987 & $(1.2617,1.8741)$ \\
\hline \multirow{2}{*}{50} & $\alpha=0.8(0.799961)$ & -0.000039 & 0.0027371 & 0.002737 & $(0.7012,0.9048)$ \\
\cline { 2 - 6 } & $\beta=1.5(1.512985)$ & 0.012985 & 0.0073598 & 0.007528 & $(1.3508,1.6777)$ \\
\hline \multirow{2}{*}{200} & $\alpha=0.8(0.800502)$ & 0.000502 & 0.0020934 & 0.002094 & $(0.7089,0.8903)$ \\
\cline { 2 - 6 } & $\beta=1.5(1.510718)$ & 0.010718 & 0.0058925 & 0.006007 & $(1.3640,16650)$ \\
\hline \multirow{2}{*}{300} & $\alpha=0.8(0.800997)$ & 0.000997 & 0.0018525 & 0.001854 & $(0.7174,0.8887)$ \\
\cline { 2 - 6 } & $\beta=1.5(1.506141)$ & 0.006141 & 0.0044795 & 0.004517 & $(1.3843,1.6418)$ \\
\cline { 2 - 6 } & $\alpha=0.8(0.800886)$ & 0.000886 & 0.0014993 & 0.00150 & $(0.7275,0.8757)$ \\
\hline \multirow{2}{*}{500} & $\alpha=1.5(1.505427)$ & 0.005427 & 0.0037086 & 0.003738 & $(1.3875,1.6335)$ \\
\cline { 2 - 6 } & $\beta=1.5(1.503156)$ & 0.003156 & 0.002423 & 0.002433 & $(1.4061,1.5991)$ \\
\hline \hline
\end{tabular}

From Tables 1, 2 and 3, we note that the Bias is reduced as the sample size is increased. 


\section{Real Data Analysis}

In this section, we illustrate the empirical importance of the OLW model and other lifetime distributions using two applications to real data.

The first data set (I): The first set consists of 63 observations of the strengths of $1.5 \mathrm{~cm}$ glass fibres, originally obtained by workers at the UK National Physical Laboratory. The data are:

$0.55,0.74,0.77,0.81,0.84,0.93,1.04,1.11,1.13,1.24,1.25,1.27,1.28,1.29,1.30,1.36,1.39,1.42,1.48,1.48,1.49$, $1.49,1.50,1.50,1.51,1.52,1.53,1.54,1.55,1.55,1.58,1.59,1.60,1.61,1.61,1.61,1.61,1.62,1.62,1.63,1.64,1.66$, 1.66, 1.66, 1.67, 1.68, 1.68, 1.69, 1.70, 1.70, 1.73, 1.76, 1.76, 1.77, 1.78, 1.81, 1.82, 1.84, 1.84, 1.89, 2.00, 2.01, 2.24. These data have also been analyzed by Smith and Naylor (1987). For this data set, we shall compare the fits of the OLW distribution with some competitive models like Weibull (W), exponential Weibull (EW), Kumaraswamy Weibull (KwW) (Cordeiro et al., 2010), beta Weibull (BW) (Lee et al., 2007), and Odd lindly Weibull with three parameters (OLW*) (Silva et al. 2016).

The second data set (II): represents 40 observations of time-to-failure $\left(10^{3} / h\right)$ of turbocharger of one type of engine, see Xu et al. (2003). The data are: 1.6, 3.5, 4.8, 5.4, 6.0,6.5, 7.0, 7.3 , 7.7, 8.0, 8.4, 2.0, 3.9, 5.0, 5.6, 6.1, 6.5, 7.1, 7.3, $7.8,8.1,8.4,2.6,4.5,5.1,5.8,6.3,6.7,7.3,7.7,7.9,8.3,8.5,3.0,4.6,5.3,6.0,8.7,8.8,9.0$. This data set is used to compare the fits of the OLW lifetime model with some competitive models like W, Lindley Weibull (LiW) (Cordeiro et al. 2018), transmuted complementary Weibull geometric (TCWG) (Afify et al. 2014) and BW models. All parameters of these distribution are positive numbers. In Tables 1 and 2, the MLEs and their standard errors (SEs) (in parentheses) of the parameters from the fitted models and the values of the Akaike Information Criterion (AIC), Cram er-von Mises $\left(W^{*}\right)$ and Anderson-Darling $\left(A^{*}\right)$ goodness-of-fit statistics are presented. According to the lowest values of the AIC, $W^{*}$ and $A^{*}$ statistics.

For the first data set, the OLW model provides the best fit. The empirical PDF and CDF for the OLW are displayed in Figures 2 and 3 respectively, which support the results of Table 4.

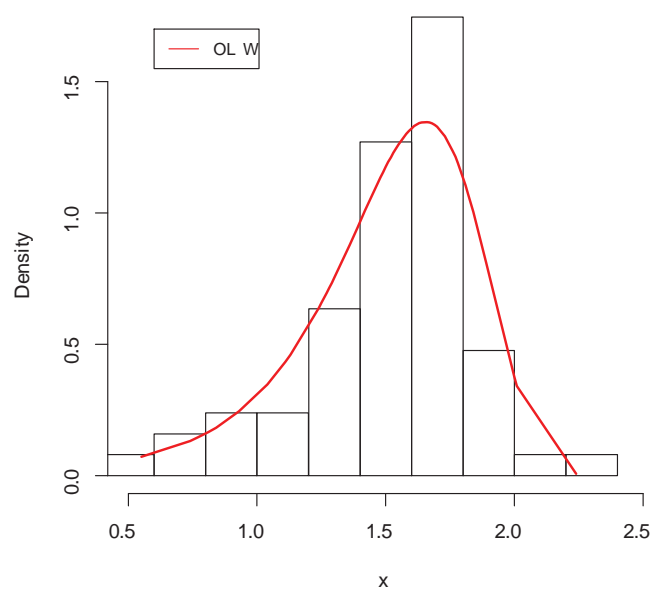

Figure 3. Estimated PDF for data set I.

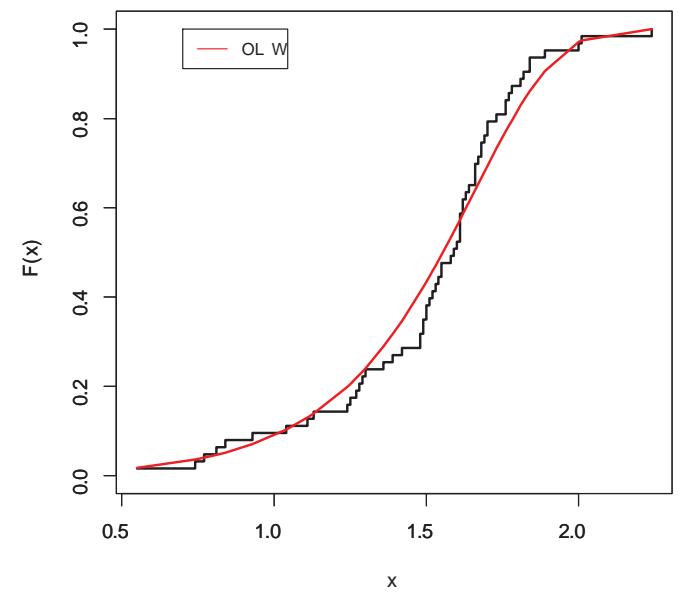

Figure 4. Estimated CDF for data set I.

For the second data set, the OLW model provides the best fit. The empirical PDF and CDF for the OLW are displayed in Figures 4 and 5 respectively, which support the results of Table 2. 


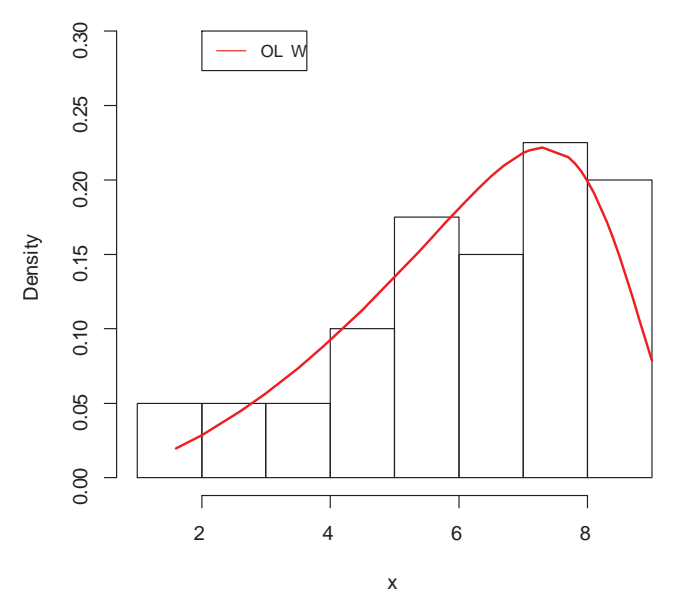

Figure 4. Estimated PDF for data set II.

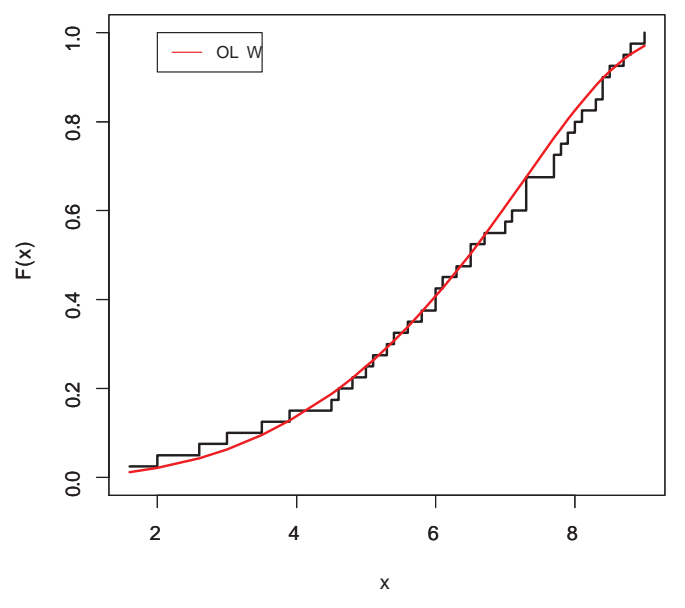

Figure 5. Estimated PDF for data set II.

Hence, we prove empirically that the proposed model provides better fits in two applications than other seven extended Weibull distributions with two, three and four parameters. There are too many models to fit and this fact really shows that the OLW model can be a good alternative for modeling survival data.

\section{Discussion}

In this work, we propose and study a new two-parameter lifetime model, called the Odd Lindley Weibull (OLW) distribution, which extends the Weibull distribution. The OLW distribution is motivated by the wide use of the Weibull model in many applied areas and also for the fact that this new generalization provides more flexibility to analyze real data. The OLW density function can be written as a linear combination of the exponentiated W (Exp-W) densities. We derive explicit expressions for the ordinary and incomplete moments, moments of the (reversed) residual life, generating functions and order statistics. We discuss the maximum likelihood estimation of the model parameters. We assess the performance of the maximum likelihood estimators in terms of biases, variances, mean squared of errors by means of a simulation study. The usefulness of the new model is illustrated by means of two real data sets. The new model provides consistently better fits than other competitive models for these data sets. The OLW lifetime model is much better than Weibull, exponential Weibull, Kumaraswamy Weibull, beta Weibull, and the three parameters Odd lindly Weibull with three parameters models so the OLW lifetime model is a good alternative to these models in modeling glass fibres data as well as the OLW lifetime model is much better than the Weibull, Lindley Weibull transmuted complementary Weibull geometric and beta Weibull models so the OLW lifetime model is a good alternative to these models in modeling time-to-failure data. We hope that the new distribution will attract wider applications in reliability, engineering and other areas of research. Finally, as a future work we will consider bivariate and multivariate extension of the OLW distribution. In particular with the copula based construction method, trivariate reduction etc. 
Table 4. The MLEs(SEs inparentheses) for some fitted models to data set $\mathbf{I}$ and the AIC, $W^{*}$ and $A^{*}$ values

\begin{tabular}{cccccccc}
\hline \hline Model & $\hat{\alpha}$ & $\hat{b}$ & $\hat{\beta}$ & $\hat{\lambda}$ & $A I C$ & $W^{*}$ & $A^{*}$ \\
\hline $\mathrm{W}$ & - & - & 5.781 & 1.628 & 34.414 & 0.237 & 1.304 \\
& - & - & $(0.576)$ & $(0.037)$ & & & \\
\hline EW & 0.671 & - & 7.285 & 1.718 & 35.351 & 0.636 & 3.484 \\
& $(0.249)$ & - & $(1.707)$ & $(0.086)$ & & & \\
\hline TW & - & -0.5010 & 5.1498 & 0.6458 & 34.6720 & 1.0358 & 0.1691 \\
& - & $(0.2741)$ & $(0.6657)$ & $(0.0235)$ & & & \\
\hline OLLW & - & 0.9439 & 6.0256 & 0.6159 & 36.3736 & 1.2364 & 0.2194 \\
& - & $(0.2689)$ & $(1.3478)$ & $(0.0164)$ & & & \\
\hline BW & 0.620 & 10.249 & 7.759 & 2.382 & 37.179 & 0.196 & 1.089 \\
& $(0.248)$ & $(95.117)$ & $(2.023)$ & $(2.897)$ & & & \\
\hline KWW & 0.606 & 0.214 & 6.908 & 1.337 & 35.252 & 0.161 & 0.908 \\
& $(0.162)$ & $(0.029)$ & $(0.004)$ & $(0.003)$ & & & \\
\hline OLW & 0.049 & - & 1.102 & 0.492 & 34.387 & 0.153 & 0.870 \\
& $(0.087)$ & - & $(0.527)$ & $(0.494)$ & & & \\
\hline OLW & 0.2026 & - & 1.716 & - & $\mathbf{3 3 . 4 2 7}$ & $\mathbf{0 . 1 3 8}$ & $\mathbf{0 . 8 1 3}$ \\
& $(0.0317)$ & - & $(0.087)$ & - & & & \\
\hline \hline & & & & & & & \\
\hline
\end{tabular}

Table 5. The MLEs(SEs inparentheses) for some fitted models to data set II and the AIC, $W^{*}$ and $A^{*}$ values

\begin{tabular}{cccccccc}
\hline \hline Model & $\hat{\alpha}$ & $\hat{b}$ & $\hat{\beta}$ & $\hat{\lambda}$ & \multirow{2}{*}{$A I C$} & $W^{*}$ & $A^{*}$ \\
\hline $\mathrm{W}$ & - & - & 3.872 & 6.920 & 82.48 & 0.0769 & 0.5730 \\
& - & - & $(0.517)$ & $(0.294)$ & & & \\
\hline LiW & - & 0.898 & 0.169 & 3.499 & 81.89 & 0.0636 & 0.4815 \\
& - & $(1.093)$ & $(0.073)$ & $(0.633)$ & & & \\
\hline TCWG & 0.188 & $-8.9 \times 10^{-5}$ & 0.2059 & 2.7881 & 81.32 & 0.0496 & 0.3766 \\
& $(0.046)$ & $(0.647)$ & $(0.2747)$ & $(0.8733)$ & & & \\
\hline BW & 0.075 & 11.242 & 0.240 & 115.43 & 79.04 & 0.0210 & 0.1696 \\
& $(0.030)$ & $(3.850)$ & $(0.102)$ & $(489.0)$ & & & \\
\hline OLW & 8.309 & - & 0.188 & - & $\mathbf{7 7 . 9 0}$ & $\mathbf{0 . 0 1 5 9}$ & $\mathbf{0 . 1 0 1 8}$ \\
& $(0.148)$ & - & $(0.046)$ & - & & & \\
\hline \hline
\end{tabular}

\section{Acknowledgements}

The authors are thankful to the Editor and the reviewers for their constructive comments and suggestions which greatly improved the current version.

\section{References}

Afify, A. Z., Cordeiro, G. M., Yousof, H. M., Abdus, S., \& E. M. M. (2016). The Marshall-Olkin additive Weibull distribution with variable shapes for the hazard rate. Hacettepe Journal of Mathematics and Statistics, forthcoming.

Afify, A. Z., Nofal, Z. M., \& Butt, N. S. (2014). Transmuted complementary Weibull geometric distribution. Pak. J. Stat. Oper. Res., 10, 435-454.

Afify, A. Z., Nofal, Z. M., Yousof, H. M., El Gebaly, Y. M., \& Butt, N. S. (2015). The transmuted Weibull Lomax distribution: properties and application. Pak. J. Stat. Oper. Res., 11, 135-152.

Alizadeh, M., Ghosh, I., Yousof, H. M., Rasekhi, M., \& Hamedani G. G. (2017a). The generalized Odd generalized exponential family of distributions: properties, characterizations and applications. J. Data Sci. 15, 443-466.

Alizadeh, M., Lak, F., Rasekhi, M., Ramires, T. G., Yousof, H. M., \& Altun, E. (2017b). The Odd log-logistic Topp Leone $G$ family of distributions: heteroscedastic regression models and applications. Computational Statistics, forthcoming. 
Alizadeh, M., Yousof, H. M. Rasekhi, M., \& Altun, E. (2018). The Odd loglogistic Poisson-G Family of distributions. Journal of Mathematical Extensions, forthcoming.

Aryal, G. R., \& Tsokos, C. P. (2011). Transmuted Weibull distribution: a generalization of the Weibull probability distribution. European Journal of Pure and Applied Mathematics, 4, 89-102.

Aryal, G. R., Ortega, E. M., Hamedani, G. G., \& Yousof, H. M. (2017a). The Topp-Leone Generated Weibull distribution: regression model, characterizations and applications. International Journal of Statistics and Probability, 6, 126-141.

Aryal, G. R., \& Yousof, H. M. (2017b). The exponentiated generalized-G Poisson family of distributions. Economic Quality Control, forthcoming.

Alizadeh, M., Merovci, F., \& Hamedani, G. G. (2015). Generalized transmuted family of distributions: properties and applications. Hacettepa Journal of Mathematics and Statistics, forthcomig.

Brito, E., Cordeiro, G. M., Yousof, H. M., Alizadeh, M. \& Silva , G. O. (2017). Topp-Leone Odd Log-Logistic Family of Distributions. Journal of Statistical Computation and Simulation, 87(15), 3040-3058.

Cordeiro, G. M., Afify, A. Z., Yousof, H. M., Cakmakyapan, S., \& Ozel, G. (2018). The Lindley Weibull distribution: properties and applications. Anais da Academia Brasileira de Ciências, forthcoming.

Cordeiro, G. M., Yousof, H. M., Ramires, T. G., \& Ortega, E. M. M. (2018). The Burr XII system of densities: properties, regression model and applications. Journal of Statistical Computation and Simulation, 88(3), 432-456.

Cordeiro, G. M., Ortega, E. M., \& da Cunha, D. C. C. (2013). The exponentiated generalized class of distributions. Journal Data Sci., 11, 1-27.

Cordeiro, G. M., Ortega, E. M., \& Nadarajah, S. (2010). The Kumaraswamy Weibull distribution with application to failure data. Journal of the Franklin Institute, 347, 1399-1429.

Cordeiro, G. M., Ortega, E. M., \& Silva, G. O. (2012). The Kumaraswamy modified Weibull distribution: theory and applications. Journal of Statistical Computation and Simulation, 1-25.

El-Bassiouny, A. H., Medhat, E., Abdelfattah, M., \& Eliwa, M. S. (2016). Mixture of exponentiated generalized WeibullGompertz distribution and its applications in reliability. J. Stat. Appl. , 5(3), 1-14.

El-Bassiouny, A. H., Medhat, E., Abdelfattah, M., \& Eliwa, M. S. (2017). Exponentiated generalized Weibull-Gompertz distribution with application in survival analysis. J. Stat. Appl. Pro., 6(1), 7-16

Ghitany, M. E., Al-Hussaini, E. K., \& Al-Jarallah, R. A. (2005). Marshall-Olkin extended Weibull distribution and its application to censored data. Journal Applied Statistics, 32, 1025-1034.

Hamedani G. G. Yousof, H. M., Rasekhi, M., Alizadeh, M., \& Najibi, S. M. (2018a). Type I general exponential class of distributions. Pak. J. Stat. Oper. Res., forthcoming. Mathematics. forthcoming.

Hamedani G. G. Rasekhi, M., Najibi, S. M., Yousof, H. M., \& Alizadeh, M., (2018b). Type II general exponential class of distributions. Pak. J. Stat. Oper. Res., forthcoming.

Khan, M. N. (2015). The modified beta Weibull distribution. Hacettepe Journal of Mathematics and Statistics, forthcoming.

Khan, M. S., \& King, R. (2013). Transmuted modified Weibull distribution: a generalization of the modified Weibull probability distribution. European Journal of Pure and Applied Mathematics, 6, 66-88.

Korkmaz, M. C., Yousof, H. M., \& Hamedani, G. G. (2017). The exponential Lindley Odd log-logistic G family: properties, characterizations and applications. Journal of Statistical Theory and Applications, forthcoming.

Lai, C. D., Xie, M., \& Murthy, D. N. P. (2001). Bathtub-shaped failure rate life distributions. pages 69-104

Lai, C. D., Xie, M., \& Murthy, D. N. P. (2003). A modified Weibull distribution. IEEE Transactions on Reliability, 52, 33-37.

Lee E. T., \& Wang J. W. (2003). Statistical Methods for Survival Data Analysis. 3rd ed.,Wiley, NewYork.

Mead, M. E., \& Afify, A. Z. (2016). On five-parameter Burr XII distribution: properties and applications. South African Statistical Journal, Forthcomig.

Mudholkar, G. S., \& Srivastava, D. K. (1993). Exponentiated Weibull family for analyzing bathtub failure-real data. IEEE Transactions on Reliability, 42, 299-302. 
Mudholkar, G. S., Srivastava, D. K., \& Freimer, M. (1995). The expnentiated Weibull family: a reanalysis of the busmotor-failure data. Technometrics, 37, 436-445.

Mudholkar, G. S., Srivastava, D. K., \& Kollia, G. D. (1996). A generalization of the Weibull distribution with application to the analysis of survival data. Journal of the American Statistical Association, 91, 1575-1583.

Murthy, D.N.P., Xie, M., \& Jiang, R. (2004). Weibull Models. 1st ed., John Wiley \& Sons, Hoboken, NJ.

Nadarajah, S. (2009). Bathtub-shaped failure rate functions. Quality and Quantity, 43, 855-863.

Nadarajah, S., \& Kotz, S. (2005). On some recent modifications of Weibull distribution. IEEE Trans. Reliab., 54, 561-562.

Nadarajah, S., \& Kotz, S. (2006). The exponentiated type distributions. Acta Applicandae Mathematica, 92, 97-111.

Nofal, Z. M., Afify, A. Z., Yousof, H. M., \& Cordeiro, G. M. (2017). The generalized transmuted-G family of distributions. Communications in Statistics-Theory and Methods, 46, 4119-4136.

Nofal, Z. M., Afify, A. Z., Yousof, H. M., Granzotto, D. C. T., \& Louzada, F. (2016). Kumaraswamy transmuted exponentiated additive Weibull distribution. International Journal of Statistics and Probability, 5, 78-99.

Rinne, H. (2009). The Weibull distribution. Chapman \& Hall, London.

Shaw, W. T., \& Buckley, I. R. C. (2007). The alchemy of probability distributions: beyond Gram-Charlier expansions and a skew-kurtotic-normal distribution from a rank transmutation map. Research report.

Silva, G. O., Ortega, E. M. M., \& Cordeiro, G. M. (2010). The beta modified Weibull distribution. Lifetime Data Analysis, $16,409-430$.

Silva, F. S., Percontini, A., de Brito, E., Ramos, M. W., Venancio, R., \& Cordeiro, G. M. (2017). The Odd Lindley-G Family of Distributions.Austrian Journal of Statistics, 46(1), 65-87.

Xie, M., Tang, Y., \& Goh, T. N. (2002). A modified Weibull extension with bathtub failure rate function. Reliability Engineering and System Safety, 76, 279-285.

Xie, M., \& Lai, C. D. (1995). Reliability analysis using an additive Weibull model with bathtub-shaped failure rate function. Reliability Engineering and System Safety, 52, 87-93.

Yousof, H. M., Afify, A. Z., Alizadeh, M., Butt, N. S., Hamedani, G. G., \& Ali, M. M. (2015). The transmuted exponentiated generalized-G family of distributions. Pak. J. Stat. Oper. Res., 11, 441-464.

Yousof, H. M., Afify, A. Z., Alizadeh, M., Nadarajah, S., Aryal, G. R., \& Hamedani, G. G. (2017a). The MarshallOlkin generalized-G family of distributions with Applications. Communications in Statistics-Theory and Methods, forthcoming.

Yousof, H. M., Afify, A. Z., Cordeiro, G. M., Alzaatreh, A., \& Ahsanullah, M. (2017b). A new four-parameter Weibull model. Journal of Statistical Theory and Applications, forthcoming.

Yousof, H. M., Afify, A. Z., Hamedani, G. G., \& Aryal, G. (2016). The Burr X generator of distributions for lifetime data. Journal of Statistical Theory and Applications, 16, 288C305.

Yousof, H. M., Alizadeh, M., Jahanshahiand, S. M. A., Ramires, T. G., Ghosh, I., \& Hamedani G. G. (2017). The transmuted Topp-Leone $\mathrm{G}$ family of distributions: theory, characterizations and applications. Journal of Data Science, 15, 723-740.

Yousof, H. M., Altun, E., Ramires, T. G., Alizadeh, M., \& Rasekhi, M. (2018). A new family of distributions with properties, regression models and applications. Journal of Statistics and Management Systems, 21(1), 163-188.

Yousof, H. M. Majumder, M., Jahanshahi, S. M. A., Ali, M. M., \& Hamedani G. G. (2017c). A new Weibull class of distributions: theory, characterizations and applications. Communications for Statistical Applications and Methods, forthcoming.

Yousof, H. M., Rasekhi, M., Afify, A. Z., Alizadeh, M., Ghosh, I., \& Hamedani G. G. (2017d). The beta Weibull-G family of distributions: theory, characterizations and applications. Pakistan Journal of Statistics, 33, 95-116. 


\section{Copyrights}

Copyright for this article is retained by the author(s), with first publication rights granted to the journal.

This is an open-access article distributed under the terms and conditions of the Creative Commons Attribution license (http://creativecommons.org/licenses/by/4.0/). 